\title{
Principles of epistemological accountability with methodological implications for measuring, assessing, and profiling human resilience
}

\author{
Astier M. Almedom $^{1,2,3}$. David O'Byrne $^{1}$ and $\underline{\text { Anne Jerneck }}^{1}$
}

\begin{abstract}
We propose two fundamental principles of epistemological accountability with critical methodological implications for studies designed to measure, assess, and/or profile human psychosocial resilience. Firstly, researchers involved in human psychosocial resilience studies owe it to the individuals and communities that they engage to disclose their motives and possible misreadings of the situations they enter, albeit with good intentions. Secondly, researchers and those individuals researched need to share a language of colearning and coproduction, and utilization of knowledge that is mutually intelligible. Again, the onus is on researchers and their funders to respect the researched and their particular epistemological sovereignties. As the number of published examples of authentic community- and/or needs-driven research and action to strengthen human psychosocial resilience increases, the sustainability of human social well-being and harmony may also be expected to rise. Psychosocial resilience encompasses a dynamic multidimensional set of personal capabilities as well as social and material assets/resources that individuals, families, and communities mobilize to mentally and emotionally embrace "turbulent" change and transformation while maintaining routine functioning without loss of identity, integrity, or core purpose in life that defines them as who they are individually as well as collectively. These proposed informed predictions are yet to be widely adopted and applied in the new paradigm for advancing this century of human psychosocial resilience, well-being, and sustainability.
\end{abstract}

Key Words: community resilience; epistemological accountability; human resilience; psychosocial well-being, sustainability studies

\section{INTRODUCTION}

Do not judge me by my successes, judge me by how many times I fell down and got back up again. (Nelson Mandela).

Resilience, a word originating from the Latin resilire meaning to rebound, is now a perennial key word for our turbulent, unpredictable, transformative 21st century. Inevitably, its definitions and meanings are still contested among researchers and practitioners alike (Almedom and Glandon 2007, Bonanno 2012), sometimes for reasons unrelated to the pursuit of scientific knowledge and understanding. As a concept and metaphor, resilience lends itself to multiple dimensions of human development, social organization, and the biosphere, so it is futile to seek to establish a single definition. Although there are many detractors, interest in the topic continues to grow. The majority of scholars and practitioners are genuinely looking to unravel the realities of human resilience in relation to sustainability of livelihoods, lifestyles, human well-being, and the biosphere on which all of these depend.

In this article, we draw attention specifically to human psychosocial resilience as documented in public discussions published in the form of peer-reviewed journal special issue, special feature, or special section collections of articles. Peerreviewed journal special issues represent concerted efforts to draw attention to a topic or theme of wide appeal to a broad spectrum of academic and other readers ranging from students and researchers to practitioners and policy makers. We focus on the meaning of the term from the standpoint of those whose psychosocial resilience has been under study. Two questions served as navigation tools in sorting the literature: (1) How may transdisciplinary researchers effectively measure, assess, and/or profile psychosocial resilience in ways that are epistemologically accountable to the individuals or communities being researched and methodologically/scientifically sound at the same time? (2)
How may scientific reports of psychosocial resilience become accessible and useful to the study participants and the communities and biosocial ecosystems in which they are nested?

Psychosocial resilience encompasses a dynamic multidimensional set of inner personal resources as well as external social and material assets/resources that individuals, families, and communities mobilize to mentally and emotionally embrace "turbulent" change and transformation while simultaneously maintaining routine functioning without loss of identity, integrity, or core purpose in life that defines them as who they are individually and collectively. The above quoted words of the late South African President Nelson Mandela, a global icon of human resilience, hint at a self-aware, highly remarkable capacity to resile, i.e., to rebound psychosocially, to resume and complete his extraordinarily long and arduous journey to freedom. Counting the number of times he "fell down and got back up again" was clearly important to Mandela; and what constituted falling down could only be determined accurately by himself. What were the instances of falling down and getting back up again that were meaningful to him, and why? Counting is easy as long as it is clear what it is that counts and is to be counted. The process (falling down and getting back up again) and not merely the outcome (success) of resiling are part and parcel of what makes a person resilient. A number of scholars have debated the question of human resilience as process versus outcome without directly engaging their human subjects in their analyses and argumentations. We propose that systematic measurements and/ or assessments of human resilience need to be grounded in the shared understanding of diverse meaning systems that influence process, outcome, and emergent narratives of the researched subjects in their natural settings, as opposed to laboratory settings where experimentally induced distress responses are analyzed and documented. 
Table 1. Principles of epistemological accountability and methodological coherence when measuring, assessing, and/or profiling human resilience.

\begin{tabular}{|c|c|c|}
\hline Theme & $\begin{array}{l}\text { Journal Special Issue/ } \\
\text { editor/s and/or proponents }\end{array}$ & Epistemological Accountability Principles; methodological implications and strategies \\
\hline Commu & $\begin{array}{l}\text { and the global mental health movement } \\
\text { Transcultural Psychiatry } 2012 \text { 49(3-4). } \\
\text { - Campbell and Burgess (Guest editors); } \\
\text { - Fernando (Contributor); }\end{array}$ & $\begin{array}{l}\text { - Context specific and place-based research: Human resilience, a deep pervasive } \\
\text { strength, considered to be easiest to measure in the context of specific risks, } \\
\text { opportunities, and social pressure. } \\
\text { - Pointed out the pitfalls of a decontextualized definition of "community" as "patients } \\
\text { and their families," and a view of community/lay primary health workers "largely as } \\
\text { handmaidens of biomedical expertise," offering services that are to be "scaled up." } \\
\text { - Advocated for social theory to guide the agenda, and not biomedicine whose citations } \\
\text { of the "human rights of the mentally ill" and "political will" for scaling up may be } \\
\text { somewhat shallow and disingeneous. } \\
\text { - Argued that the Diagnostic and Statistical Manual of Mental Disorders published by } \\
\text { the American Psychiatric Association was developed using symptoms observed and } \\
\text { documented in Caucasian American patients. Exporting them to other countries to be } \\
\text { used "rather unquestioningly among people very different from Caucasian Americans" } \\
\text { remains problematic. }\end{array}$ \\
\hline
\end{tabular}

Community psychology and global climate change American Journal of Community Psychology 201147 (3-4).

- Reimer and Reich (editors);
- "Whose empowerment do we facilitate and with whom do we need to raise critical consciousness about the interconnections of global climate change with social justice, poverty, and capitalism: Those most vulnerable to the impacts of global climate change or those who create the most damage?

- How does the concept of sense of community apply when the need to act together goes beyond the boundaries of local communities and the members of communities change because of immigration?

- What version of community should we strive toward, if the current one is part of the problem?" (p. 351).

Psychology's contributions to understanding and addressing global climate change American Psychologist 2011 66(4).

- A call for innovation, acute awareness of the complexities that pose serious epistemological and methodological challenges for psychologists and others engaged in

- Swim et al. (editors) interdisciplinary and transdisciplinary research collaborations across scales.

Affirmative challenges in indigenous resilience research Pimatisiwin: A Journal of Aboriginal and Indigenous Community Health 2008 6(2).

- Lessons learned from the "failure to ask the right questions" related to culture and spirituality in earlier studies helped to improve the research tools and analysis in subsequent studies.

- Anderson (Guest editor)

- Anderson and Ledogar (Contributors);

- "Aboriginal concerns drove the research, Aboriginal communities were users of the evidence, and researchers were Aboriginal... strategies for returning the evidence to the communities for discussion and action became more effective" (p. 67).

\section{METHODS}

This synthesis is part of a larger systematic literature review initiated during 2013-2014 to assess the lay of the land in transdisciplinary thinking around measuring, assessing, and profiling human resilience among scholars and practitioners alike. The first author conceptualized the study and provided guidelines for processing and analyzing the content of peer-reviewed journal articles retrieved from multiple databases including ISI, Web of Knowledge (Web of Science), PubMed, Scirus, SCOPUS, EMBASE, MEDLINE, and PsychInfo. The third author reviewed earlier drafts of the manuscript and provided critical social science perspectives, and the second author assisted in the process of literature survey and review.

All items indexed or catalogued in one or more of the above databases by the first quarter of 2013 were first surveyed using search terms such as community OR social AND resilience, psychosocial AND resilience, sense of coherence AND salutogenesis OR resilience, climate change OR natural disasters AND resilience. No date limits were imposed on the searches. Usually, different journals get their content indexed in databases with varying speeds, depending on their subscriptions or investments in databases. Exclusion bias arising from possible time lag between publication and indexing dates was controlled for in part by surveying additional collections and stand-alone articles captured by author-specfic and/or keyword-specific citation alerts generated by PubMed (National Center for Biotechnology Information), SCOPUS, and some individual journals. Direct contact with authors known to have relevant publications in press also yielded additional items up until July 2014. Peer-reviewed journal special issues focusing on human, psychosocial, and/or community resilience and community development were hand-sifted, and those directly or indirectly addressing issues of meaning and methods were short-listed for indepth analysis. 
Non-English language publications could not be included. However, particular attention was paid to the varying uses of the English language within and between academic disciplines, as well as health policy and practice sectors. For instance, the term "ecological" is widely used in the health sciences to mean population level or community level, as opposed to individual level or unit of analysis. It has a different meaning in environmental sciences and ecology, of course, where "ecological" refers to the natural environment and ecosystems and "socialecological" relates to interactions between environment and communities or societies. Similarly, the word "community" has multiple meanings and uses depending on the word that follows or precedes it, including community and/or societal resilience, rural community, community of practice, international community, and so forth.

\section{RESULTS}

Initially, 111 journal special issues, sections, and features/themes were retrieved, the majority of which represented a steady increase in interest on climate change alone $(n=63)$. Others looked generally at resilience to climate-related disasters $(n=17)$ and other "natural disasters" $(n=6)$, community development and resilience $(n=10)$, and social-ecological resilience, governance, and learning $(\mathrm{n}=5)$. These were hand-sifted for content of editorials and/or contributions explicitly or implicitly addressing epistemological and/or methodological issues. Surprisingly few did so with reference to human/psychosocial resilience.

The journal themes and disciplinary/interdisciplinary/transdisciplinary background of authors introducing and/or contributing to special issue and special sections were highly diverse. For the most part, each volume or section, and even some of the contributed articles, presented stand-alone deliberations with little or no crossreferencing between contributing authors. This is a common shortcoming because contributing authors do not usually read each other's drafts, although editors may in some cases share the contributed abstracts in advance. The majority of special issues on climate change did not concern themselves with psychosocial well-being as much as they did with infrastructure, organizational, institutional (governance), economic, and/or ecosystem resilience. We used two criteria for identifying the materials to be included in our analysis and synthesis:

1. Articles that concerned themselves with epistemological and methodological questions in mental health research in general, and in human resilience studies in particular.

2. Articles that concerned themselves with the challenges of knowledge coproduction and/or accessibility of research reports and with new insights on psychosocial resilience by the study participants themselves and their communities.

Key epistemological accountability principles were distilled both from stand-alone articles within special issue/section/feature sets and from other unrelated seminal works. The top four journal special issues identified as contributing fundamental principles are presented in summary form (see Table 1) and discussed in detail below.

\section{DISCUSSION}

Mental health and human resilience research and practice have been undergoing a paradigm shift "from vulnerability to strength" in recent years (Almedom and Tumwine 2008). The World Health Organization's division of mental health and the humanitarian affairs section formally adopted a global agenda (WHO 2013, see Overview) to reform mental health services to promote "overall well-being, functioning, and resilience of individuals, societies, and countries recovering from emergencies." However, social research in which the researched subjects fully engage in coproduction and utilization of knowledge and insights is yet to be recognized and integrated in epistemologically accountable and methodologically coherent ways in the Western biomedical Global Mental Health Movement agenda.

According to the editorial in a special issue of the journal Transcultural Psychiatry by social psychologists Catherine Campbell and Rochelle Burgess (2012:380), a background meeting was convened for the purposes of forging "a role for social scientists, with particular emphasis on the need for frameworks of analysis and action to clarify and expand the role of communities in advancing the Movement's agenda" [emphasis added]. While acknowledging the top-down nature of the movement, the special issue editors were ambivalent about how local communities with their own mental health competence may engage with the movement on ethically acceptable and equitable terms. They pointed out some of the salient points of the Global Mental Health Movement agenda, which has been set by a dominant international coalition of agencies and actors.

Gaithri Fernando's (2012) contribution to the same special issue illustrated the points made by the editorial. Herself a psychologist, Fernando illustrated her main argument by presenting the results of her quick search of PychINFO database (a database where predominantly psychology journals are indexed/catalogued) using the phrase "research agenda" as a search term with and without adding the term "culture." She found a more than $90 \%$ decrease in yield when culture was added, indicating to her that the vast majority of publications of research agenda ignored culture. However, the questions, "Who speaks for the community?" and "Whose interpretation of the knowledge counts (the researcher's or the researched)?" are not adequately addressed in this special issue. Epistemological sovereignty of the discipline, in this case psychology, is implicit in the discussions, which are pitched at getting the dominant Western biomedical paradigm to give some leeway (see Table 1 for a summary).

The following year, a noteworthy study conducted by Sri Lankans in Sri Lanka was published in the International Journal of Mental Health Systems. Daya Somasundaram and Sambasivamoorthy Sivayokan (2013) reported on a qualitative, ecological study of community resilience in postwar northern Sri Lanka. They examined and documented psychosocial risk and protective factors at the level of individual, family, and community. The multigenerational family unit and spiritual dimension of Tamil culture and traditions were highlighted as central to understanding the lived experience of ordinary people in the aftermath of the long war that had spanned three decades. Although the authors combined sociological and psychological introspection and phenomenology (as outsiders) in their critical enquiry to understand their study participants' daily predicaments, they also saw their research as "autoethnographic." They identified themselves as ethnic Tamils (insiders), examining critically their own communities. Their earlier findings on how 
the creative arts became "valuable conduits for the expression of emotions, finding meaning and developing meaningful community narratives" during the war and after the Tsunami are cited in their recommendations for psychosocial programs that they promote as "important for restoring well-being and functioning, but also to rebuild social capital and community resilience which would enable and empower affected communities to help themselves" (Somasundaram and Sivayoka 2013).

Manuel Reimer and Stephanie Reich (2011:351), editors of a special section of the American Journal of Community Psychology on Community Psychology and Global Climate Change, emphasized the need for community psychologists to be involved in global climate change research, arguing that "a comprehensive discussion of how the topic of global climate change relates to the core theoretical frameworks in community psychology" was missing. They asked three interconnected questions concerning researchers' ethical and epistemological accountability (see Table 1). However, the questions were not developed further or addressed by other contributors to the special section.

American Psychologist, the flagship journal of the American Psychological Association, also published an agenda-setting special issue on the topic of global climate change in the same year (Swim et al. 2011) with an introduction presenting a detailed analysis of the role of psychologists in illuminating the human causes and consequences of climate change. One of the contributions mentioned ethical concerns associated with global inequalities in energy consumption that bedevil the global discourse on climate adaptation, mitigation, and response to climate change-induced disasters.

At this point, it is worth noting that very few studies of human resilience have so far based their enquiry on epistemological premises that originate from expressed views and expectations of their study populations, i.e., the populations that were being researched. This has arguably resulted in a "crisis of identity" in psychiatry, a branch of medicine that specializes in mental health. According to Patrick Bracken, a leading practitioner in the field of transcultural psychiatry:

... One of the most important difficulties is around the
perennial question of what is an appropriate
epistemology for psychiatry. What sort of knowledge can
we have with regard to mental illness and what sort of
expertise is possible?... Debates about epistemology are
not simply an intellectual exercise. Many psychiatrists
feel that they cannot be "real doctors" unless their
discipline is grounded in the naturalscience epistemology
that guides the rest of medicine... [But] natural science
methods reach their limits in the territory of mental
health and illness. This is largely a territory of meanings,
values and relationships.... If we are to be truly
"evidence-based" in our discipline, we need a radical
rethinking of our guiding epistemology: a move from
reductionism to hermeneutics. (Bracken 2014:241).

In a special issue of Pimatisiwin: A Journal of Aboroginal and Inidgenous Community Health, an open-access electronic journal that is also available in print for readers who have no access to the Internet, focusing on resilience in youth, Neil Andersson and Robert Ledogar (2008) reflected on the lessons learned from earlier Canadian Aboriginal community-based research that was supported by the Centro de Investigaciones de Enfermedades Tropicales (CIET). CIET originated at the Universidad Autonoma de Guerrero, Mexico, in 1986 with Dr. Neil Andersson as its Science Director, offering intensive training in epidemiology to health research and service delivery planners and researchers from Mexico and many different countries that focused on engaging diverse, marginalized indigenous populations. Andersson and Ledogar (2008:66) espoused iterative processes of colearning and coproduction of knowledge using a "strong and evolving conceptual framework of resilience" that focused on Aboriginal "resilience rather than on pathologies." The ethical review and approval for the research involved several different Aboriginal/First Nation review panels, and the Health Canada ethics review board in cases in which two senior scientists from the Public Health Agency of Canada were involved as coinvestigators. This approach is different from that adopted by researchers in Australia, where youth mental health "expert panels" drawn from the Aboriginal communities themselves may be recruited to serve in consensus-forming processes using the Delphi method (see, for instance, Chalmers et al. 2014).

In both his editorial introductory and concluding statements, Andersson observed that "the positive tone of resilience research changes the terms of engagement between researchers and the communities. Almost everyone is interested to learn about their strengths. This is good research" (Andersson 2008a:3). Furthermore, pointing out the subtle distinction between indigenous resilience and research into indigenous resilience, he concluded that:

\begin{abstract}
Measurement offers glimpses of resilience, mostly from the potentially distorted view of how resilient youth face specific adversity - adversity that is set by the funding opportunity: tobacco, substance abuse, suicide, or HIV infection. The driving role of funding has obvious problems; the priorities of the funders may not be the priorities of communities and results can tell more about the funding opportunity than about resilience itself. Even so, this problem-focused research has the very practical advantage of producing results geared to solutions. A major lesson of this body of work is that we should allow ourselves the space (and the modesty) to recognize that Aboriginal resilience is greater than we have been able to measure under specific funding opportunities.
\end{abstract} (Andersson 2008b:201).

\section{Measuring human psychosocial resilience}

Measurement is a critical requirement in scientific research as well as practice, particularly in mental health, emotional well-being, and psychosocial resilience, in which it demands ethical and epistemological justification first and foremost (Mirowsky and Ross 2002, Andersson 2008b). In their seminal paper, Mirowsky and Ross, prominent medical sociologists, had argued most persuasively that measurement is essential for linking concepts with the lived realities of human subjects of research as they experience and understand their own realities. In their own words, "Measurement is our one hope of escaping preconception. Without it we cannot distinguish shared observations from shared beliefs" (Mirowsky and Ross 2002:152). These authors promoted the use of an index or indexes consisting of open questions, with 
responses scored on a scale that each respondent would rank. They strongly advised against the use of simplified reductionist diagnostic checklists consisting of questions that are designed to elicit mere yes or no answers. According to Mirowsky and Ross, diagnostic tools that consist of a predetermined number of symptoms or clusters of symptoms commonly grouped together on an ad hoc basis, with the cut-off points set by experts, often distort the respondents' lived realities. Clinical diagnoses that rely on checklists hide the full range of feelings and experiences of the individual, and mask differences, disparities, and possible shared meaning systems of the communities or societies in which individual lives are nested.

The problems associated with the checklist method of diagnosing mental and emotional dis-ease are many. Mirowsky and Ross highlighted three: Firstly, it is expert oriented rather than client centered and it puts the control over human suffering in the hands of professionals rather than of clients. It positions the professional's implicit stance along the lines of "This is over your head. Put yourself in my hands" (Mirowsky and Ross 2002:154). Secondly, a checklist often refers to discrete and mutually exclusive entities (absent or present) rather than to a multifaceted spectrum of symptoms, and thus polarizes clients into limited categories. Thirdly, it uses medical/technical language that may not be accessible or helpful to assist clients in their predicament. By contrast, an index allows ample room for respondents to participate meaningfully based on their own realities and experiences, as long as the researchers permit it, in the process of data gathering. For example, as conveyed in Mandela's words, the instances of falling down and getting back up again may be the preference as to how the researched individuals may wish to be judged. Thus, knowledge generated by an index or indexes may be by design potentially more easily accessible and useful to the researched. If the study participants become active participants in the study design from the outset, the research process and results can inform and empower them to take control of their own mental health care needs and minimize their risk of being labeled and/or socially stigmatized by diagnoses assigned to them by mental health experts. Such knowledge may also assist mental health policy makers and practitioners in the design and implementation of effective and sustainable preventive as well as curative actions. Both the process and outcome of such research would be likely to contribute directly to sustainable mental and emotional well-being across the life course, and in turn to the psychosocial resilience of successive generations.

Although a diagnosis requires certainty in terms of whether a subject has a dis-ease or not, an index seeks reliability in terms of how well emotions and cognitions can be assessed, described, represented, and understood, as well as acted upon. Indexes that cover not only the type but also the degree, frequency, intensity, and severity of a problem or condition are therefore superior tools for assessing psychological conditions (Mirowsky and Ross 2002). By serving as a link between the felt emotion (reality) and the analytical concept (theory), an index allows subjects to assess and express what they feel or experience in relation to a condition while also allowing the researcher to measure and interpret the symptoms in relation to a scale or social frame (Mirowsky and Ross 2002).

How emotions and cognitions are manifested, described, and represented matters for how we understand and act upon them.
Following Mirowsky and Ross (2002), we argue that a human science should aim to produce knowledge that is not only useful for people but also made available to them (Mirowsky and Ross 2002). Any epistemological effort to create a human science should therefore start by recognizing and building on the knowledge that people themselves produce when they talk about their experiences, feelings, and perceptions as subjects of social research in health care settings (Mirowsky and Ross 2002). This can be achieved by asking semistructured questions that bring to light, rather than mask, the richness of personal accounts and narratives. Qualitative approaches using research strategies such as case study, ethnography, grounded theory, narrative design, and phenomenology may all be used as modes of inquiry to serve the purpose of advancing empirically grounded, methodologically sound, and theoretically informed knowledge on how individuals and communities resile.

In line with the above argument, Almedom et al.'s studies of human resilience published between 2003 and 2013 present a case in point. Originally developed in response to displaced Eritrean women respondents' suggestions to researchers to ask "better questions" focusing on how they "survived and even thrived against the odds," the studies resulted in the innovative use of an index, the Sense of Coherence (SOC-13) scale, which was modified for use by scoring on a 5-point Likert scale. The SOC-13 was adapted to reflect multilingual Eritrean sensibilities because it was administered in 9 languages (see Almedom et al. 2005 and Almedom and Glandon 2007). As the study findings were published, the pre-existing processes of community organizing on the ground had come to fruition, successfully mobilizing both bonding and bridging types, and cognitive as well as structural forms of social capital with the goal of enabling the internally displaced to return to their homes (see Nayr et al. 2011). Almedom's team reports reflect both insiders' and outsiders' understanding of the lived realities of the study participants. Almedom et al.'s epistemologically accountable approach influenced the choice and combination of methods of investigation and analysis used.

Moreover, unlike Andersson et al.'s studies of community resilience in Canada conducted over a number of years, the Eritrean studies were inclusive of all sections of the population: rural and urban; highland, lowland, and mid-altitude ecologyoriented livelihoods; and most importantly, all ethnic and language groups. The wider context and background in which the country Eritrea had successfully laid strong foundations for all citizens to actively participate in nation building on equal terms under its policy of Unity in Diversity. This facilitated the process of colearning and knowledge coproduction. The adapted and modified index (SOC-13) became the Sense and Sensibilities of Coherence method, which combined ethnographic qualitative and quantitative techniques of investigation, analysis, and dissemination of results (see Almedom 2004, 2011, Almedom et al. 2005, Almedom and Glandon 2007, Nayr et al. 2011, Parker et al. 2013).

\section{Principles of epistemological accountability}

Human psychosocial resilience studies inevitably entail intertwined epistemological and methodological issues that are not uncommon in other fields of resilience science and policy research and practice; see, for example, Miller et al. (2008) and Vogel et al. (2007), respectively, for their cogent discussions of 
epistemological pluralism in transdisciplinary research and the multiplicity of theories of knowledge that play out in the sciencepolicy interface. Miller et al.'s treatise on the inevitable "epistemological sovereignty" issues that bedevil relationships between stakeholders in interdisciplinary studies is highly relevant to human resilience studies. Similarly, the spider webs of interactions between science and policy that Vogel et al. address in some depth pertain to the researched with respect to their role in knowledge coproduction and/or their entitlements to access and utilization of the knowledge. However, neither include the psychosocial dimensions of human resilience, so our analysis provides insights that may instigate new thinking and connecting opportunities for readers of Ecology and Society and related scientific journals to pursue.

Epistemological pluralism has been proposed to advance interdisciplinarity in resilience science (Miller et al. 2008), and sustainability science advocates methodological pluralism while also striving for transdiciplinarity (Jerneck and Olsson 2011). Our findings call for more explicit epistemological and methodological considerations in human resilience research informed by the lessons learned: namely, that it is important to focus resilience research on individual and collective assets and strengths instead of needs and weaknesses (otherwise, the research would be no different from vulnerability research following the old paradigm); that resilience research should be participatory, involving the researched communities in all stages of the process, including the evaluation and use of the research findings; and sociocultural parameters should be at the center of human resilience research instead of at, or outside, the margins of biomedically and funding needs-driven studies.

\section{CONCLUSIONS}

At its core, the question of measuring human resilience is an inherently ethical one. It is clear that generation of knowledge that is neither accessible nor useful to those involved in research as the sources of that knowledge would run counter to human resilience and sustainability of psychosocial well-being at any level: individual or collective.

One form of neglect of the researched is particularly evident. A prevalent mechanistic framing sees human resilience as something that, when lacking, can be introduced to the situation, whether individual or community. In this case, measurement of resilience is based on a universal model that understands the concept as something that can be "diagnosed" as present or absent, with the implication that if it is found to be lacking, it can then be imposed. This is destined to deliver alienation through overly reductionist epistemologies, which neglect the experience of those being researched. Other understandings see resilience as an emergent yet universal human potential that can be drawn, to a greater or lesser extent, from almost any situation of adversity. Measurement in this framing can accommodate the many different courses that human resilience can run, but is always grounded in the common substrates of individual and collective human cognition and structures and is of greater use to those who live the experience. Perhaps this epistemological shift can complement the discursive paradigm shift from vulnerability to resilience, which has thus far largely entailed the repackaging of an old model.

In conclusion, based on our synthesis, we propose the following two fundamental principles of epistemological accountability with important methodological implications for measuring, assessing, and profiling human resilience. Firstly, researchers involved in human psychosocial resilience studies owe it to the individuals and communities that they engage to disclose their motives and possible misreadings of the situations they enter, albeit with good intentions. Unless they actively seek to understand the lived realities of their subjects and openly acknowledge them as the source of information and insights to be applied to sustainable solutions to human psychosocial predicaments, the researchers will exacerbate human suffering by imposing epistemological supremacy built on sustained ignorance and possible arrogance. Many a research report has been published and disseminated by policy makers and practitioners relying on the colloquial quick and dirty surveys at huge expenses to themselves and to the researched. We urge scientists of all denominations, i.e., natural, social, behavioral, interdisciplinary, and transdisciplinary, to take the time and care necessary to meaningfully engage their human subjects and to provide trustworthy reports for funding bodies and policy makers to use in their decision making on resource allocation.

Secondly, researchers and the researched need to share a language of colearning and coproduction, and utilization of knowledge that is mutually intelligible. Again, the onus is on researchers and their funders to respect the researched and their particular epistemological sovereignties. As the number of published examples of authentic community- and/or needs-driven research and action to strengthen human psychosocial resilience increases, so would the sustainability of social well-being and harmony be expected to rise.

It may be speculated that the above two fundamental principles of epistemological accountability would foster, rather than impede (as may well be feared), high levels of activity in the form of funded research and action that are based on longer range sustainable development premises built on forward-looking terms of engagement between "expert," knowledge-bearing stakeholders. These proposed informed principles are not new or unheard of, but they are yet to be widely adopted and applied in the new paradigm for advancing this century of human psychosocial resilience, well-being, and sustainability.

\section{Responses to this article can be read online at: http://www.ecologyandsociety.org/issues/responses. $\mathrm{php} / 7313$}

\section{Acknowledgments:}

The authors thank the Rockefeller Foundation and Lund University's Centre for Sustainability Studies (LUCSUS) for funding support. Constructive comments and suggestions were gratefully received from three anonymous reviewers.

\section{LITERATURE CITED}

Almedom, A. M. 2004. Factors that mitigate war-induced anxiety and mental distress. Journal of Biosocial Science 36:445-461. http://dx.doi.org/10.1017/S0021932004006637

Almedom, A. M. 2011. Ngügï wa thiong'o: listening for peace and resilience in Africa-from Makerere 1962 to Asmara 2000. 
Pages 548-569 in W. Dietrich, editor. The Palgrave international handbook of peace studies: a cultural perspective. Palgrave McMillan, New York, New York, USA.

Almedom, A. M., and D. Glandon. 2007. Resilience is not the absence of PTSD any more than health is the absence of disease. Journal of Loss and Trauma: International Perspectives on Stress \& Coping 12(2):127-143. http://dx.doi.org/10.1080/15325020600945962

Almedom, A. M., B. Tesfamichael, Z. S. Mohammed, C. G. N. Mascie-Taylor, and Z. Alemu. 2007. Use of "sense of coherence (SOC)" scale to measure resilience in Eritrea: interrogating both the data and the scale. Journal of Biosocial Science 39(1):91-107. http://dx.doi.org/10.1017/s0021932005001112

Almedom, A. M., B. Tesfamichael, Z. S. Mohammed, J. Muller, N. Mascie-Taylor, and Z. Alemu. 2005. "Hope" makes sense in Eritrean sense of coherence, but "loser" does not. Journal of Loss and Trauma: International Perspectives on Stress and Coping 10 (5):433-451. http://dx.doi.org/10.1080/15325020500193887

Almedom, A. M., and J. K. Tumwine. 2008. Resilience to disasters: a paradigm shift from vulnerability to strength. African Health Sciences 8(S):1-4.

Andersson, N. 2008a. Affirmative challenges in indigenous resilience [editorial]. Pimatisiwin: A Journal of Aboriginal and Indigenous Community Health 6(2):3-6.

Andersson, N. 2008b. Directions in indigenous resilience research [afterword]. Pimatisiwin: A Journal of Aboriginal and Indigenous Community Health 6(2):201-208.

Andersson, N., and R. J. Ledogar. 2008. The CIET aboriginal youth resilience studies: 14 years of capacity building and methods development in Canada. Pimatisiwin: A Journal of Aboriginal and Indigenous Community Health 6(2):65-88.

Bonanno, G. A. 2012. Uses and abuses of the resilience construct: loss, trauma, and health-related adversities. Social Science \& Medicine 74:753-756. http://dx.doi.org/10.1016/j.socscimed.2011.11.022

Bracken, P. 2014. Towards a hermeneutic shift in psychiatry. World Psychiatry 13(3):241-243. http://dx.doi.org/10.1002/wps.20148

Campbell, C., and R. Burgess. 2012. The role of communities in advancing the goals of the movement for global mental health. Transcultural Psychiatry 49(3-4):379-395. http://dx.doi. org/10.1177/1363461512454643

Chalmers, K. J., K. S. Bond, A. F. Jorm, C. F. Kelly, B. A. Kitchener, and A. J. Williams-Tchen. 2014. Providing culturallyappropriate mental health first aid to an Aboriginal or Torres Straight Islander adolescent: development of expert consensus guidelines. International Journal of Mental Health Systems 8(6).

Fernando, G. A. 2012. The roads less traveled: mapping some pathways on the global mental health research roadmap. Transcultural Psychiatry 49(3-4):396-417. http://dx.doi. org/10.1177/1363461512447137

Jerneck, A., and L. Olsson. 2011. Breaking out of sustainability impasses: how to apply frame analysis, reframing, and transition theory to global health challenges. Environmental Innovation and Societal Transitions 1:255-271. http://dx.doi.org/10.1016/j. $\underline{\text { eist.2011.10.005 }}$
Miller, T. R., T. D. Baird, C. M. Littlefield, G. Kofinas, F. S. Chapin, III, and C. L. Redman. 2008. Epistemological pluralism: reorganizing interdisciplinary research. Ecology and Society 13 (2): 46. [online] URL: http://www.ecologyandsociety.org/vol13/ iss $2 / \operatorname{art} 46 /$

Mirowsky, J., and C. E. Ross 2002. Measurement for a human science. Journal of Health and Social Behavior 43(2):152-170. http://dx.doi.org/10.2307/3090194

Nayr, A, S. Noor, and A. M. Almedom. 2011. Community organizing to end displacement in Eritrea: a narrative of community and institutional resilience. Pages 81-93 in W. Rathke, editor. Global grassroots: an organizing perspective. Social Policy Press, New Orleans, Louisiana, USA.

Parker, J., L. J. Tiberi, J. Akhilgova, F. Toirov, and A. M. Almedom. 2013. "Hope is the engine of life"; "hope dies with the person": analysis of meaning making in FAO-supported North Caucasus communities using the "Sense and Sensibilities of Coherence" (SSOC) methodology. Journal of Loss and Trauma: International Perspectives on Stress \& Coping 18(2):140-151.

Riemer, M., and S. M. Reich 2011. Community psychology and global climate change: introduction to the special section. American Journal of Community Psychology 47(3-4):349-353. http://dx.doi.org/10.1007/s10464-010-9397-7

Somasundaram, D., and S. Sivayokan. 2013. Rebuilding community resilience in a post-war context: developing insight and recommendation - a qualitative study in Northern Sri Lanka. International Journal of Mental Health Systems 7(3). http://dx. doi.org/10.1186/1752-4458-7-3

Swim, J. K., P. C. Stern, T. J. Doherty, S. Clayton, J. P. Reser, E. U. Weber, R. Gifford, and G. S. Howard, editors. 2011. Psychology and global climate change. American Psychologist 66(4):241-250.

Vogel, C., S. C. Moser, R. E. Kasperson, and G. D. Dabelko. 2007. Linking vulnerability, adaptation, and resilience science to practice: pathways, players, and partnerships. Global Environmental Change 17(3-4):349-364. http://dx.doi.org/10.1016/ j.gloenvcha.2007.05.002

World Health Organization. 2013. Building back better: sustainable mental health care after emergencies. World Health Organization, Geneva, Switzerland. 